\title{
Fixing Sickness Care Component of Comprehensive Primary Health Care in India
}

\section{Suresh $\mathrm{K}^{*}$ \\ Public Health Consultant, India}

*Corresponding author: Suresh Kishanrao, Public Health Consultant \& Visiting ProfessorMPH, Schools of Environmental Science, Public Health and Sanitation Management, Karnataka State Rural Development and Panchayat Raj University (KSRDPRU), GADAG and Rajiv Gandhi Institute of Public Health, Bengaluru, Karnataka .Tel: 918029571102, 919810631222; Email: ksuresh.20@gmail.com

\section{Research Article}

Volume 3 Issue 3

Received Date: May 11, 2020

Published Date: June 18, 2020

DOI: $10.23880 /$ jqhe-16000165

\section{Abstract}

With recent Coronal virus Pandemic India, hopefully public health in general and comprehensive primary health care will come to the forefront. The focus will shift from private hospitals to government hospitals as private hospitals are too small to handle large-scale health emergencies and care of the poor. And instead of sourcing medical devices and equipment and drugs from abroad, everything will be sourced from India. In February 2017, Government of India promised to upgrade 150,000 existing HSCs and PHCs into Health and Wellness Centres (HWCs) that will switch from "selective" to "comprehensive primary health care" and start providing the larger package of services in Public Sector Health.

Empowering paramedical workers for early diagnosis and dispensing pre-decided drugs began way back in 1953 for Malaria control. The training of Multipurpose Health Workers (Male and Female) introduced capacity building for minor ailments treatment in 1974. Later vertical interventions at community level, like Control of diarrheal diseases (1980), Acute Respiratory infections (1990), standalone new-born care and integrated management of neonatal and childhood illnesses (IMNCI) in early 2000 were added in the job descriptions of the health workers. NHP 2017 makes it mandatory to run outpatient clinics for 6 hours every day at the HWCs, institutionalizing regular sickness care services at the community level for comprehensive health care.

The design of HWCs and the delivery of services build on the experiences and lessons learnt from the National Health Mission, India's flagship programme for strengthening health systems. To address the expanded service delivery package will require reorganization of work processes, addressing the continuum of care across facility levels; moving from episodic pregnancy and delivery, new born and immunization services to chronic care services; instituting screening and early treatment programmes; ensuring high-quality clinical services; using information and communications technology, focusing on health promotion and addressing health literacy.

The announcement of HWCs received wide coverage and attention. However, promises are an integral part of Indian polity; the challenge is implementation as witnessed being part of the health system by the author since 1968. The first Health and Wellness Centre (HWC) under Ayushman Bharat was inaugurated by the Prime Minister at on 14/04/2018. Since then 29214 AB-HWC are reported functional as on 30.01.2020. The word functional is used for issuing government order or at the most change of the sign board of the facility. Two years down the line there is hardly any visible change in the strategy of 
providing sickness care for six hours a day as envisaged and starting of the new interventions included in service package on the ground level. The availability of services would evolve in different states gradually, depending on three factors- a) the availability of suitably skilled human resources at the HWC, b) the capacity at district/sub-district level to support the HWC in the delivery of that service, and c) the ability of the state to ensure uninterrupted supply of medicines and diagnostics at the level of HWC.

This article analyses the existing primary health care system in the country, challenges of establishing HWCs for CPHC specially at HSC level and the way forwards.

Keywords: Comprehensive Primary Health Care; Health Sub-centre; Health and Wellness Centres; Middle Level Health Provider; Universal Health Coverage

Abbreviations: CPHC: Comprehensive Primary Health Care; UHC: Universal Health Coverage; HSCs: Health Sub Centres; PHCs: Primary Health Centres; CHCs: Community Health Centres; HWCs: Health Wellness Centres; MLHP: Middle Level Health Provider; NHP: National Health Policy; MOH\&FW; Ministry of Health \& Family Welfare; AB: Ayushman Bharat; ASHA: Accredited Social Health Activist; AWW: Anganwadi Worker; HA(M\&F): Health Assistants (Male \& Female); MO: Medical Officer.

\section{Introduction}

After the first case of Corona Virus on 31 January 2020, the pandemic has shaken the health system in India. The full effects of the pandemic are yet to be measured, but change is already here to see. For first time ever in the history of Public Health India showed that it was a whole-of-society's approach to health that aimed at equitably to maximize the level and distribution of health and well-being focusing on people's needs and preferences early in the pandemic and ensuring the continuum from health promotion and disease prevention efforts like, containment, social distancing, hand wash, use of masks, cough etiquettes, toilet hygiene to treatment, rehabilitation and lockdown, and as close as feasible to people's everyday environment. Focus shifting to strengthening government health system by creating dedicated facilities, procuring ventilators, PPEs, and Rapid test kits and strengthening laboratory services for PCR.

We are now convinced that Public health in general and comprehensive primary health care will come to the forefront. People must learn to live with SARC Cov-2 (Covid 19) and many other viruses and pathogens. The focus will shift from private hospitals to government hospitals as private hospitals are too small to handle large-scale health emergencies and care of the poor. Instead of sourcing medical devices and equipment and drugs from abroad, everything will be sourced from India.
The concept of PHC has been repeatedly reinterpreted and redefined in the years since 1978, the most recent being in October 2018, in Astana, Kazakhstan, when world leaders met to commemorate the $40^{\text {th }}$ anniversary of the 1978 Declaration. This vision places people, as individuals and communities, as the central focus of all efforts towards PHC. People's fundamental right to the highest attainable standard of health and well-being, and the world's renewed commitment to social justice, are expressed through adequate social protection and concerted efforts to address the needs of those who are most disadvantaged. Comprehensive Primary Health care (CPHC) now is considered as a whole-of-society approach to health that aims equitably to maximize the level and distribution of health and well-being by focusing on people's needs and preferences (both as individuals and communities) as early as possible along the continuum of care from health promotion and disease prevention to treatment, rehabilitation and palliative care, and as close as feasible to people's everyday environment [1].

\section{CPHC Has Three Inter-Related and Synergistic Components}

1. Meeting people's health needs through comprehensive promotive, protective, preventive, curative, rehabilitative, and palliative care throughout the life course, strategically prioritizing key health care services aimed at individuals and families through primary care and the population through public health functions as the central elements of integrated health services; 2 . Systematically addressing the broader determinants of health (including social, economic and environmental factors, as well as individual characteristics and behaviour) through evidence- informed policies and actions across all sectors; and 3. Empowering individuals, families, and communities to optimize their health, as advocates for policies that promote and protect health and well-being, as codevelopers of health and social services, and as self-carers and caregivers. This new approach to 
Comprehensive primary health care is central to achieving the SDGs and UHC. Progress will require courage and determination, but the time is right [1].

\section{Constitutional Provisions, Evolution and Challenges for Comprehensive Primary Health Care in India}

The Constitutional provisions (Schedule 7 of article 246) are classified into three lists, including a Concurrent list which both centre and states can govern but the overriding power is with the centre. Central List (list includes original numbers): 28. Port quarantine, including hospitals connected therewith; seamen's and marine hospitals 55. Regulation of labour and safety in mines and oilfields State List: 6. Public health \& sanitation; hospitals \& dispensaries 9. Relief for disabled \& unemployable Concurrent List: 16. Lunacy and mental deficiency, including places for the reception or treatment of lunatics and mental deficient 18. Adulteration of foodstuffs and other goods. 19. Drugs and poisons, subject to the provisions of entry 59 of List I with respect to opium 20A. Population control and family planning 23. Social security and social insurance; employment and unemployment. 24. Welfare of labour including conditions of work, provident funds, employers' liability, workmen's compensation, invalidity and old age pensions and maternity benefits 25 . Education, including technical education, medical education and universities, subject to the provisions of entries 63, 64, 65 and 66 of List I; vocational and technical training of labour.] 26. Legal, medical and other professions 30.Vital statistics including registration of births and deaths [2].

In India concept of primary healthcare was laid down by the recommendations of Bhore Committee as early as 1946. Bhore committee report laid emphasis on social orientation of medical practice and high level of public participation. With beginning of health planning in India and first five-year plan formulation (1951-1955) Community Development Programme was launched in 1952. It was envisaged as a multipurpose program covering health and sanitation through establishment of primary health centres (PHCs) and sub-centres. By the close of second five-year plan (19561961) Health Survey and Planning Committee (Mudaliar Committee) recommended to limit the population served by the PHCs with the improvement in the quality of the services provided and provision of one basic health worker per 10,000 population. The Jungalwalla Committee in 1967 gave importance to integration of health services. The committee recommended the integration from the highest to lowest level in services, organization, and personnel. The Kartar Singh Committee on multipurpose workers in 1973 laid down the norms about health workers. Shrivastav Committee (1975) suggested creation of bands of paraprofessionals and semi-professional worker from within the community like schoolteachers and post-masters. It recommended the development of referral complex by establishing linkage between PHCs and high-level referral and service centres (First Referral Units- FRUs). Rural Health Scheme was launched in 1977, wherein training of community health, reorientation training of multipurpose workers, and linking medical colleges to rural health was initiated. To initiate community participation, the community health volunteer "Village Health Guide" scheme was launched. The Alma-Ata Declaration of 1978 launched the concept of health for all by year 2000. Alma-Ata declaration led to formulation of India's first National Health Policy in 1983. The major goal of policy was to provide universal, comprehensive primary health services. Nearly 20 years after the first policy, the second National Health Policy was presented in 2002. The National Health Policy, 2002 set out a new framework to achieve public health goals in socioeconomic circumstances currently prevailing in the country. All five-year plans had overly ambitious plans but fell short of the set targets and never were a corrective measure taken. In last 7 decades of independence, we have seen much improvement in primary healthcare services, infrastructure, and related healthcare indices of the country. Still many challenges are ahead to achieve health for all [3]. Realizing the need to review primary healthcare in the country to know our strengths and weaknesses to face the challenges in the future, India has committed for Comprehensive Health care in the National Health Policy 2017 to achieve Sustainable Development Goals (SDGs) through Universal Health Coverage (UHC) [4].

\section{Current National Health Policy 2017}

National Health Policy 2017 [4] is in place with a Goal of the attainment of the highest possible level of good health and well-being, through a preventive and promotive health care orientation in all developmental policies, and universal access to good quality health care services without anyone having to face financial hardship as a consequence. Public Health care services consist of preventive, promotive, curative and rehabilitative services. For the first time in Indian Public Health history NHP 2017 has committed for providing at least 6 hours OPD case management in 150,000 Health and Wellness Centres (HWCs) across the country. Health and Wellness Centres are envisaged to deliver an enlarged range of services to address the primary health care needs of the entire population in their area, expanding access, ensuring universality and equity close to the community. These services in HWCs will be provided through a Mid-level Health Care Provider (MLHP)/Community Health Officer (CHO) placed at each HWC-SHC and Medical Officer at PHC (Rural/Urban).

As on 31st March 2019, there were 157411 Health Sub Centres (HSCs), 24855 Primary Health Centres (PHCs) 
and 5335 Community Health Centres (CHCs) in rural areas functioning in the country [5]. The HWC at the sub health centre level would be equipped and staffed by an appropriately trained Primary Health Care team, comprising of Multi-Purpose Workers (male and female) \& ASHAs and led by a Mid-Level Health Provider (MLHP). Together they will deliver an expanded range of services encompassing reproductive and child health services, communicable diseases, non-communicable diseases, palliative care and elderly care, oral health, ENT care, and basic emergency care4. A Primary Health Centre (PHC) that is linked to a cluster of HWCs would serve as the first point of referral for many disease conditions for the HWCs in its jurisdiction. The Medical Officer at the PHC would be responsible for ensuring that CPHC services are delivered through all HWCs in her/his area and through the PHC itself. The number and qualifications of staff at the PHC would continue as defined in the Indian Public Health Standards (IPHS). Majority of secondary and tertiary care facilities in public sector will also provide primary health care. Apart from public sector facilities there are thousands of private sickness care providers both formal including Allopathic, Ayurveda, Yoga, Unani, Siddha Homeopathy systems and other traditional and informal service providers.

\section{Burden of Illness in Rural and Urban India}

As per NSSO 75th round report (2017-18), the proportion of persons that responded as ailing (PPRA) was $7.5 \%$ of the $(9.1 \%$ among urban and $6.8 \%$ among rural) population during last 15-day reference period of the survey [6]. Gender wise PPRA indicated the proportion to be around $6.7 \%$ male in and in female-8.3\%. The PPRA ranged between less $3 \%$ in Assam and Bihar to a highest of $24.5 \%$ in Kerala. While six states of MP, Karnataka, Chhattisgarh, Rajasthan, Telangana, Haryana had the PPRA in the range of 3-5.9 \%. Another six namely Tamil Nadu, Gujarat, Jharkhand, UP, Maharashtra, Odisha had a PPRA of 6-8.9\% respectively. Punjab, West Bengal, Andhra Pradesh had the PPRA of 10.014. Age specific ailment reporting was highest of $27.7 \%$ among persons aged 60 years and above followed $11.4 \%$ among $40-59$ years, $8.5 \%$ among under-fives, 5.4\% among 20-39, 4.8\% among 5-14 years and least among 15-29 aged$3.3 \%$. Similar trend was observed among both genders.

Broad category of illnesses (Figure 1) indicates that both in Urban (35.7\%) and rural (25.4\%) area infections were dominant, followed by cardiovascular diseases. While metabolic disease in urban areas were almost double compared to rural area. It is also evident that the proportion of CVDs and Metabolic diseases was significantly higher in urban areas.

\author{
A. Existing Services to continue: \\ 1. Care in pregnancy and childbirth. \\ 2. Neonatal and infant health care services. \\ 3. Childhood and adolescent health care services. \\ 4. Family planning, Contraceptive services and other \\ Reproductive Health Care services. \\ 5. Management of Communicable diseases including \\ National Health Programmes. \\ 6. Integrated Disease Surveillance \\ B. Expanded services to be initiated at Community: \\ 7. Daily Outpatient care for acute simple \& minor \\ ailments. \\ 8. Screening, Prevention, Control and Management \\ of Non-Communicable diseases. \\ 9. Care for Common Ophthalmic and ENT problems. \\ 10. Basic Oral health care. \\ 11. Elderly and Palliative health care services. \\ 12. Emergency Medical Services. \\ 13. Screening and Basic management of Mental \\ health ailments.
}

Figure 1: Range of services.

The average medical expenditure incurred per spell of ailment indicated that, people spend from INR 327 (Govt.) to 1081 in Private hospitals, the private clinics (564) and Trust hospitals cost 624 . The breakup of the components on which the expenditure was made indicated that most of the money was spent on Medicines (54-82\%), followed by Diagnostics (11-29\%), Doctor's fee and others. In the Govt. sector first 2 items costed $93 \%$ and the doctor's fee- $15 \%$ in pro-profit facilities.

The inclination towards seeking sickness care was clearly highest towards allopathic treatment as over $95 \%$ in both the sectors sought allopathic care. Less than 5\% sough care in AYUSH system facilities. More than 65\% (62\% in rural and $71.5 \%$ in urban) spells of ailment were treated in the private. Govt. /Public facilities catered only $32.5 \%$ and 26.2 $\%$ in rural and urban areas respectively.

An overall 29 persons (Urban-34 and Rural 26) per 1000 people fell sick sought hospitalization (in 365 days). Kerala reported the highest proportion 105/1000. Hospitalization proportion was 111/1000 among persons aged over 70 years, followed by 72 in 60-69 years and 27 among underfives. As I am looking at primary health care in this paper, I am not detailing the care of hospitalized.

Health Insurance/assurance coverage was poor as $81 \%$ 
of urban population and $86 \%$ in Rural Population was not covered by any health insurance scheme. Percentages of persons in different quintile class of household having some form of health expenditure coverage ranged from 10\%$22 \%$ in Rural India and from $10-33 \%$ in Urban India, $4^{\text {th }}$ and fifth quintiles in urban area showing better insurance coverage. Among the covered $13.5 \%$ in Rural and $12.2 \%$ in Urban area were covered by Govt. funded insurance scheme. In urban areas only $3.8 \%$ by households had insurance with companies, $2.9 \%$ by employer funded schemes and remaining $0.2 \%$ by others. The contribution of these three insurance schemes was only $0.6 \%$ in rural area.

Overall percentage of persons with disability in the population was 2.2 percent [7] (with $2.3 \%$ in rural and 2 $\%$ in urban areas) during July 2018 to December 2018 in the country, as per a National Statistical Office (NSO) survey report on 23 November 2019 [6]. Locomotor disabilities dominate with a share of $1.1 \%$ of the population, followed by hearing disability of $0.3 \%$, visual disability, speech and language disability and mental retardation or intellectual disability $(0.2 \%$ each) and $0.1 \%$ each of mental illnesses and other disabilities. All these needs to be considered for planning Comprehensive Primary health care services.

\section{Why Comprehensive Primary Health Care Facilities matter}

Healthcare facilities are the basic building blocks of a health system. A healthcare facility is designed to provide a certain type of services based on the size of the local population and epidemiological pattern of the health conditions of the area.

a. Public Sector Primary health care set up (Figure 2) in India provide the essential preventive and curative care required to address the most prevalent conditions, including reproductive and maternal health, child health, nutrition and diagnostic and treatment services for most common conditions. It is also from these PHC facilities that we run public health programs and communitybased programs using community health workers like \{Accredited social health activists (ASHA's) Anagnwadi workers (AWWs), Traditional Birth (TBAs) Attendants and other community volunteers\}. NHP 2017 brought about one another initiative of bringing all AYUSH health facilities that were running as vertical facilities until 2013 getting integrated strategically Under NHM at the primary and secondary care level facilities recently. The process is on and yet to see total integration across the country.

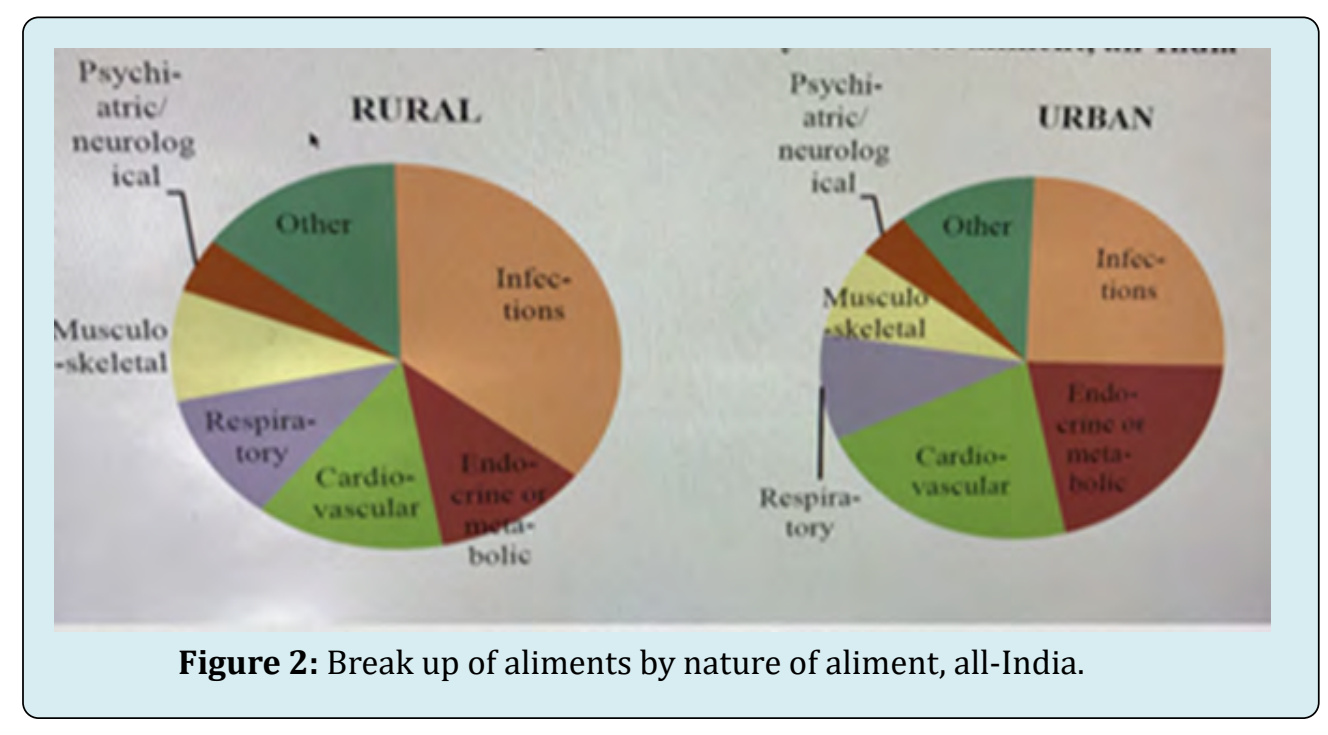

The patients with less frequent or serious conditions that require specialized diagnostic and treatment services are referred to secondary care (sub-district \& district) hospitals where more complex medical technology can be accessed and basic specialists like Physicians, surgeons, Paediatricians and Obstetrician and Gynaecologists provide services. Specialized hospitals that deliver tertiary and even quaternary levels of care for trauma, cancer, burns, cardiac surgeries etc. are in state capitals and some other big cities depending on geographic accessibility. In an extraordinary decision Govt. of India has made provision to hire such services from the market if they are not available in the nearest public health facility under National Health Protection Scheme popularly known as Ayushman Bharat $[7,8]$.

b. Public Sector Health Services in Urban areas: Primary Health Care for urban areas was not given its 
due importance since independence. Many vertical programs did have a component of urban planning and were implemented through multiple agencies like Urban Health and Family Welfare centres, Post-partum centres under All India Postpartum Program, Urban Malaria program, Urban Leprosy units, Tuberculosis program, HIV/AIDs etc. The sickness care was traditionally provided to the urban poor population through municipal dispensaries, urban PHCs and clinics. Supplementary preventive and promotive health services are also available through School Health Clinics, Bal wadi's and urban ICDS centres run by the State Women and Child Welfare Departments. Most of the secondary and tertiary facilities like maternal and child health hospitals (run by Municipal Administration state Health \& Family Welfare department) Central Government Health Services (CGHS), Employees Sate Insurance Scheme (ESIC) Railways hospitals and Defence hospitals and infectious diseases hospitals also provide primary health care for their select clientele. Government of India with the help of soft loan from the World Bank under Health system strengthening projects between mid- 1980's to 2000 established urban health posts, Primary Health centres and referral hospitals in major cities like Mumbai, Delhi, Kolkata, Chennai, Bengaluru and Hyderabad, that are under the administrative control of local Municipal corporations. Health service delivery challenges include i) Skewed distribution of public health facilities leading to lack of coverage ii) Lack of urban health promotion towards better access iii) Multiplicity of Service Provides and lack of Coordination \& Convergence (among Govt. of India/State Government/ Municipal Corporation / Municipalities and NGO sector) iv) Inadequate policy \& infrastructural capacity to deal with swelling migrant and urban poor populations.

After the launch of National Health Mission in 2013 [816], a formal Primary health care structure was set up (Figure 3) for Universal Health Coverage (UHC). They are coming up in all categories of towns \& cities, though the take-off has been slow across the country. Structural Problems currently being addressed include a) Catchment areas for the specific health facilities are not well defined b) Inadequate focus on Primary health care facilities c) Governance-Fragmentation of health care services under different government departments and local bodies and Low use of technology for data collection and integration of services. Equally challenging are the solutions such as revamp the existing facilities with ensuring one "primary urban health centre with outreach and referral facilities', rationalizing urban primary health structure, introduction of a public health management cadre and health systems management cadre, strong regulation, accreditation, and supervision framework and partnership with nongovernmental providers for closing gaps in health delivery gaps in the urban sector.

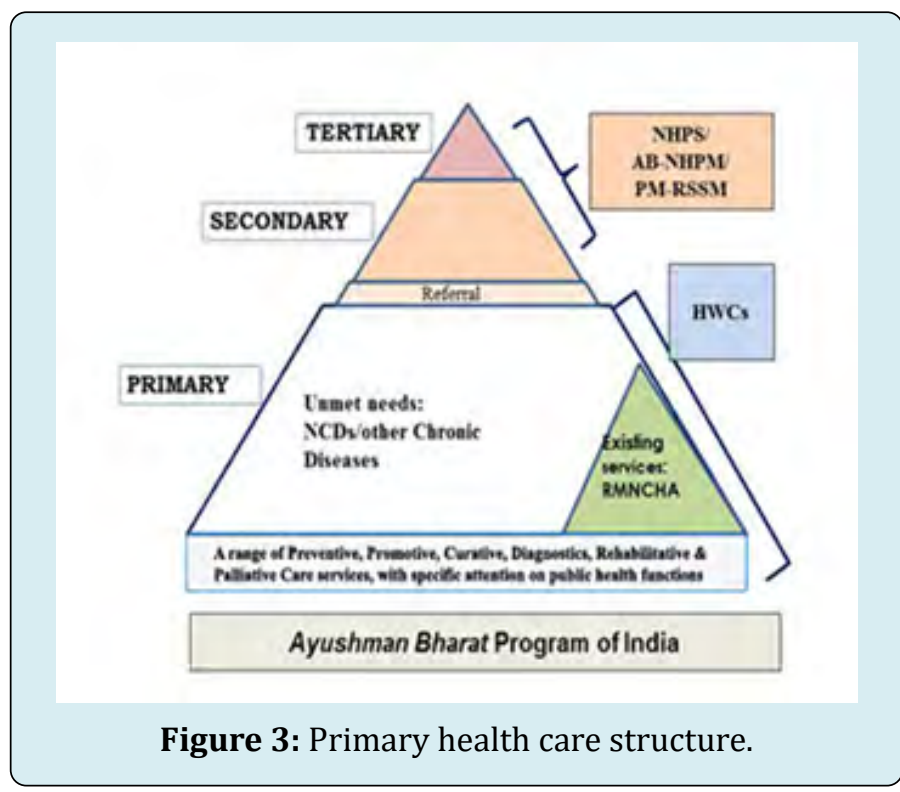

\section{Private Sector}

i) Rural Private Health Sector: The apathy of public doctors leads to unregulated private practitioners in health sector. Many of them are quacks, who work for 6 months to one year with a qualified doctor and then start their own independent practice often rendering services in the houses Figure 4. Countrywide less than $26 \%$ (U)-32\% (U) of households depend on public facilities. Nearly $68 \%$ of rural households receive medical care from private practitioners. $42 \%$ of those classified as allopathic doctors in rural areas, have no medical training. This proliferation of unregulated and unqualified private providers demands an effective regulatory system (India Development Report, 2012/13). 80\% of general practitioners practise allopathic medicine without proper training [5].

ii) Private sector in Urban India: Urban health care services are mainly dominated by Private facilities ranging from Jhola Chhap (mobile quacks- door to door service provider) registered medical practitioner (RMP) to super-specialists. The complexity of private health care in urban areas increases as the size of the town/city increases. Host of Private health providers including Private Medical colleges, pro-profit corporate hospitals and nursing homes and clinics run by family physicians are the hallmark of the urban health services. Bigger cities will also have some Trust \& Charitable Hospitals. 


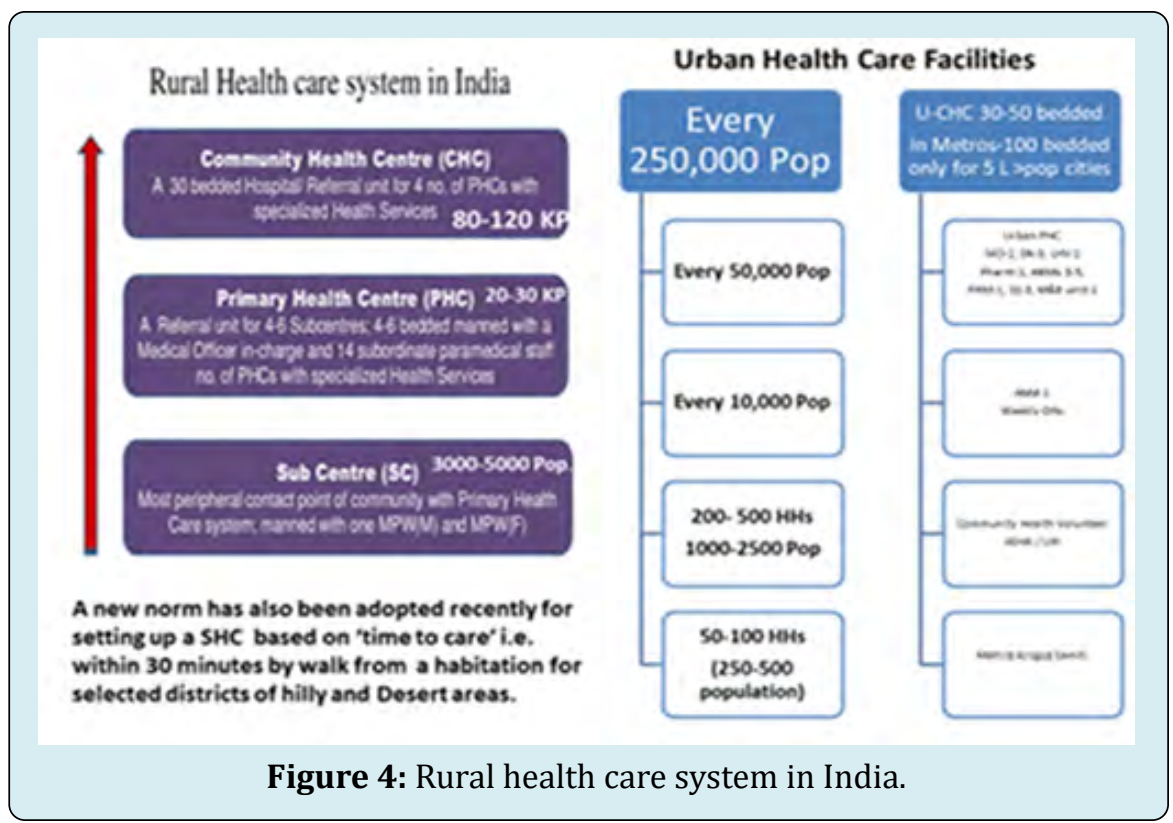

The primary care in urban areas, is dominated by the single practitioner clinics and unqualified practitioners. The markets where these providers operate are fragmented and unregulated. The quality of care is also questionable. The recent studies in most metropolitan cities and state capitals revealed that the quality of care is low in the poorer neighbourhoods. The care differed with difference in incentives (income and reputation) and competencies amongst the providers. It was also noted that rich availed the services of the more competent doctors and the poor had access to lower quality of doctors. Though the urban poor are not restricted geographically in accessing these health facilities, lack of money is a barrier to care.

Private Sector provides care to $70 \%$ of outpatient episodes of which Private Hospitals contribute (62\%), Nursing Homes (24\%), Charitable Hospitals (3\%), Corporate Hospitals (2\%), Others (1\%), In terms of service providers Medical \& Dental constitute (52\%), AYUSH Doctors (10\%), Diagnostic Labs, ISM hospitals (14\%), Nursing \& Physiotherapy (2\%) and Others (2\%) [9].

\section{The primary health care facilities matter in India health system because:}

1. The Primary care is known to place a greater emphasis on the health of the whole person rather than a specific organ or system, which may contribute to positive effects such as reduced mortality rates due to the accumulated contribution of the care.

2. The close and ongoing relationship between patients and their primary care providers helps health professionals to understand their situation more completely and make the relevant recommendations.
3. Primary health care implemented in a timely and helpful manner can also reduce the need for specialist care, which may be unnecessary and have the potential to harm patients.

4. The facilities are close to the community, thereby reduce the commuting time \& cost.

5. They are the backbone of our health system providing greater access to needed medical services; good quality of care; early management of health problems and more focus on prevention of diseases -leading to lower morbidity.

6. The Public sector UHC facilities (HWCs) as envisaged under NHP 2017 can provide free primary health care without many hassles. That will lead to many people visiting the doctor that will result in improved prices as the cost will be dependent on the value of excellent service given to a patient rather than the number of services offered to a patient

7. Primary care practitioners can recommend screening measures to detect early changes that could be indicative of specific diseases. This may include checking blood pressure, blood tests, breast examinations, mammograms, Pap smears and bowel cancer screening.

8. Most importantly they will reduce the unnecessary burden on secondary and Tertiary Hospitals, Convenience for those who need their attention and the waiting time.

9. It is in this context that this review is undertaken to analyse the challenges of making Comprehensive primary health care a reality and suggest way forwards to make the best opportunity Ayushman Bharat has provided. 


\section{Materials and Methods}

The current paper seeks to pinpoint key challenges of fixing the Comprehensive Primary Health Care, particularly Sickness Care at public sector primary health care facilities and governing private primary health care at the community level. It also discusses about the possible strategies to be taken forwards by the state and local government for overcoming the quality and cost-efficient services of the private sector. This is a review paper based on analysis of 1) secondary sources like GOI and sates portal platforms i.e. AB-HSCs progress, Stare NHM sites, 1.http://bit.ly/33QiIAk, 2. http:// bit.ly/2zdkMUV, http://alfa.nic.in/const/ schedule.html 2) NSSO reports on reported illnesses (NSSO $75^{\text {th }}$ round), Disability Report (NSSO $76^{\text {th }}$ round report), books, NGO reports, few published articles over last 50 years in journal articles, and 3) Filed assessments and observations, health facility records and interaction with stakeholders at different levels in last 15 months (September 2018-Decmeber 2019). Filed visits were made as a part my professional consultancy work that took me to 7 states, 10 districts and 5 cities in India during the said period. 4) Literature research of two MPH scholars Mr. Rashid and Ms Priyanka from KSRDPRU Gadag for their Dissertation work on a) Status of HWCs in Gadag and b) Availability of Generic lifesaving drugs in Gadag and Kushtagi, respectively Karnataka -November-December 2019.

\section{Study Setting}

Seven States across India namely Uttar Pradesh, Bihar, Madhya Pradesh, Maharashtra, Karnataka, Delhi, and Haryana. This study was taken up along with another study for UNDP supported project assessment where the author was a team leader.

\section{Sampling \& Sample Size}

Sampling of districts were done in consultation with state level officers, representing one typical and another tribal or remote district and ease of commuting for the primary objective.

\section{Geographical Scope}

The primary data collection through field level consultation was carried out in five states of Uttar Pradesh, Madhya Pradesh, Bihar, Delhi, Haryana, Maharashtra, and Karnataka covering two districts in each state and one urban area. The districts covered included Gaya and Patna in Bihar, South west and North Delhi slums, Gurgaon and Faridabad in Haryana, Bengaluru urban districts and Gadag in Karnataka, Jhabua and Bhopal in MP, Agra and Barabanki in UP. The urban areas included cities of Bengaluru (BBMP), Bhopal,
Delhi, Luck now, and Patna. In each district different level stakeholders were interviewed \{CASHA \&AWW at Villages, Health Assistants M\&F at Health Sub centres, Medical officers at PHCs and CHCs and Dy. $\mathrm{CMOH} \& \mathrm{CMOH}$ at district level and Director, joint directors at the state level). Similarly, in urban areas ASHAs, AWWS, HA's (Jr \& Sr-M \&F), MOS and municipal health officers were contacted and filed observations at Urban Health centres were made.

\section{Activity Wise Scope}

The overall focus of this study was baseline evaluation of twenty-two activities of HSS2, mostly a qualitative focus to evaluate the baseline of Health System Strengthening Support (HSS2) $2^{\text {nd }}$ project of UNDP to India, for routine Immunization system strengthening and implementation. Since the HWCs establishment was in its' initial adoption/ adaption stage we added five basic questions in interviews and observed certain functions at all levels of the health system. The study did not focus on beneficiary level feedback.

\section{Data Collection Methodology}

The primary data for this component of the study was collected by 2 methods: 1 . Questionnaire: five questions that included were i) Status of the State government order of sanctioning up gradation of HSCs \& PHCs into HWCs and if sanctioned their definition of functioning ii) If the population to be covered by such HWCs in urban areas were mapped out iii) The physical infrastructure identified, assessed for additionalities required iv) Human source recruitment and training process v) Orientation of state/district/ city and local level started 2) On site interaction with staff recruited and observation of the activities like function OPD, Antenatal clinic, laboratory services, drug dispensing, outreach services provided if any etc.

\section{Data Analysis}

Since the number of total units were only about a dozen, the data for questions were analysed in an excel sheet and the qualitative components of the functionality recorded for each of the units visited. FGD's of the staff gathered for a weekly meeting were done where possible. No video or audio-recording was done of the interviews or FGDs done during the entire process.

\section{Results}

\section{From literature Review}

The design of HWCs and the delivery of services build on the experiences and lessons learnt from the National Health Mission, India's flagship programme for strengthening 
health systems. Expanding the scope of these components to address the expanded service delivery package will require reorganization of work processes, including addressing the continuum of care across facility levels; moving from episodic pregnancy and delivery, new-born and immunization services to chronic care services; instituting screening and early treatment programmes; ensuring high-quality clinical services; and using information and communications technology for better reporting, focusing on health promotion and addressing health literacy in communities. The national and State governments did realize and spelt out clearly that although there will be major challenges ahead to meet these ambitious goals, it is important to capitalize on the current high level of political commitment accorded to comprehensive primary health care ${ }^{4}$ and strengthening public sector health care services and purchasing the services not available in public sector from the private providers under Ayushman Bharat.

The fact of the matter is NHP 2017 clearly recognized that healthcare in Rural India faces major challenges of quality, accessibility, and affordability for a large section of the population. The key limitations identified were i) shortage of qualified healthcare professionals and services like qualified doctors, nurses, technicians as evidenced by the presence of 0.76 doctors and 2.09 nurses per 1,000 people as compared to World Health organization (WHO) recommendations of 1 doctor and 2.5 nurses per 1,000 population respectively\}, and ii) infrastructure- Indian healthcare faces acute shortage of hospital beds with 1.3 hospital beds per 1,000 population as compared to WHO recommended 3.5 hospital beds per 1,000 population [9-11]. Non-uniform accessibility to healthcare across the country, physical access continuing to be the major barrier to both preventive and curative health services, and glaring disparity between rural and urban India. Most of the private facilities are concentrated in and around tier 1 and tier 2 cities, due to which patients must travel substantial distances for basic and advanced healthcare services [9].

India has a vast public health infrastructure with 23,391 primary health centres (PHCs) and 145,894 Health subcentres (HSCs)providing health services to $72.2 \%$ of the country's population living in rural areas. Although the numbers look impressive, their functional status in terms of physical infrastructure, manpower, equipment, drugs, and other logistical supplies that are greatly needed for ensuring quality services is a matter of concern. Among the key facilities which are going to be upgraded as HWCs, the status of HSCs and PHCs is not rosy as $20 \%$ of them don't have their own building, $25 \%$ do not running water supply, one third of them do not have electricity and $10 \%$ of them do not have all weather roads 6 . Sixty per cent of primary health centres (PHCs) in India have only one doctor while about five per cent have none [9]. A recent study in Nellore district of Andhra Pradesh indicated that the deficiency of AYUSH medical officers was $86.6 \%$ and the deficiency of health workers (female) was $13.33 \%$ [13]. Some of the important drugs such as antihypertensive, anticonvulsants, emergency drugs, drops, ointments, and solutions were available in less than $50 \%$ of the PHCs. Only $47 \%$ of the PHCs had Typhi-dot tests and $\mathrm{H} 2 \mathrm{~S}$ test strips to assess the water contamination, and in the labour rooms only $20 \%$ of the PHCs have a Standard Surgical Set for episiotomies in accordance with IPHS.

The first Health and Wellness Centre (HWC) under Ayushman Bharat was inaugurated by the Prime Minister Shri Narendra Modi at Jangla in Bijapur Chhattisgarh on 14thApril 2018. Since then 10,252 HWCs have been operationalized. The states of Andhra Pradesh, Tamil Nadu, Uttar Pradesh, Karnataka and Kerala lead the way with the highest number of HWCs under Ayushman Bharat. Andhra Pradesh has operationalized 1361 HWCs, Tamil Nadu 1318, UP 912, Karnataka 700 and Kerala 678 HWCs. These HWCs have been categorised as Sub Health Centres (SHC), Primary Health Centres (PHC) and Urban Primary Health Centres (UPHC). As of 11 December 2019, out of total HWCs of 39069, HSC upgraded number was 19577, PHCs upgraded were 16238 and Urban PHCs upgraded were 3254.

According to the Economic Survey 2019-20, report tabled in Parliament on Friday by Finance Minister Nirmala Sitharaman on 31 January 2020, reported that a total of 28,005 such centres have already been set up as on January 14, 2020 (source- Economic Times -31 January 2020) (Table 1).

\begin{tabular}{|c|c|c|}
\hline Description & India & Karnataka \\
\hline Footfall- & $3,10,92,556$ & 1085957 \\
\hline HT patients- treated & $69,41,364$ & 39316 \\
\hline Diabetics treated- & $38,17,736$ & 58340 \\
\hline Yoga sessions conducted & $2,03,727$ & 11628, \\
\hline Persons screened for Breast Cancer & $82,45,292$, & 20931 \\
\hline Persons screened for Cervical Cancer & $49,79,183$ & 7733 \\
\hline Persons screened for Oral Cancer & $1,22,17,040$ & 164,843 \\
\hline
\end{tabular}

Table 1: Service deliveries. 


\section{From Primary Sources \& Field Observations}

- $\quad$ All the states had issued Go's for the first set of districts to upgrade HSC s \& PHCs to HWCs

- Since most of UPHCs and HSCs in urban area were of recent origin, no further order was explicitly issued in any of the states visited. Three of the states also felt that converting HSCs into HWC's in cities is non-viable for want of physical infrastructure and difficulty in getting space for the same.

- In the UPHC the area and population to be covered was indicated, but majority had not mapped their geographical boundaries. None of the UPHC had demarked areas for ASHAs for mobilization activities and some for even Health workers for service provision

- The districts were struggling to freeze the list of HSCs and PHC for up gradation due to political pressure a common feature in India

- The most remote and needy HSC were not considered in the first lot selected for up gradation, leaving those unreached or under-reached

- The state and district level officers were oriented about the concept of HWCs either by National or Regional workshops

- Two states had frozen the qualification for MLHP (BSc.nursing), recruited and training started.

- The assessment of the identified institutions for additional inputs was not yet initiated.

- The capacity development modules for the additional tasks to be performed at the HWCs level (or better integrated modules) were in discussions at national levels only

- No concrete strategy for capacity building especially for the para-medical Primary health care providers was yet developed

- At the health sub-centres, no, new activities had yet begun, especially fulfilling the provision of running OPD for 6 hours a day.

- Paucity of Jan Aushadhalayas, that have just opened in 1-2 number per district and Taluka level for accessing lifesaving=generic drugs at reasonable cost and nearly two thirds of drugs procured for free distribution in public sector hospitals are generic drugs.

\section{Discussions}

The status of shortfall in health facilities as per 2011 population in India indicates that as against the number of PHCs required 29337 in position were 25743 and a short fall $6430(22 \%)$ and $\mathrm{CHCs}$ required were 7322 , in position 5624, shortfall 2188 (30\%) as per Rural Health Statistics (RHS), 2018, as on 31.03.2018, as per Minister of State (Health and Family Welfare) written reply in the Rajya Sabha here on 23July-2019. The number of ANMs sanctioned were 216665 and 219326 were working with a shortfall of 27964 (13\%).

As on March 31, 2018, at least 60 per cent sub centres, 18 per cent PHCs and 12 per cent CHCs in India were without staff toilets as against the WHO's mandate of basic sanitation facility at a health care centre should include at least one toilet dedicated for staff, at least one sex-separated toilet with menstrual hygiene facilities, and at least one toilet accessible for people with limited mobility. Nearly $61 \%$ govt health centres in rural India don't have separate toilets for women. In Kerala and Telangana, $86 \%$ rural health centres don't have sex-separated toilets. As on March 31, 2018, there were 26,360 sub centres and 1,313 primary health centres in rural India that did not have a regular source of water as against the WHO's recommendation of availability of sufficient quantities of safe water is important for health centres to provide quality services ${ }^{9}$. Manipur leads this list with 79 per cent sub centres devoid of regular water supply, followed by Mizoram (62\%) and Meghalaya (60\%). Among large states, Bihar has 49 per cent sub centres without regular water supply, Jharkhand (53\%), Rajasthan (34\%) and Odisha (30\%). Uttar Pradesh, Telangana, Goa and Tamil Nadu are among states where all sub centres have regular water supply.

Sub centres are the smallest units in India's rural health infrastructure under the charge of an ANM (auxiliary nurse midwife) to ensure availability of last mile trained medical services in rural areas providing all primary healthcare services. A PHC on the other hand is a government hospital with 6 beds that acts as the first contact point with local community and generally caters to around 25 villages under the charge of a qualified MBBS doctor assisted by a pharmacist, 4-5 nurses and other medical staff. PHCs treat patients with routine illness and are also equipped to handle delivery cases, organise sterilisation camps etc. A CHC is a much bigger hospital, generally with 30 beds and 5 medical experts, including a surgeon and more than 10 nurses. A CHC covers nearly 120 villages.

NHP 2017 has incorporated 7 Key Policy Shifts. Of them four are clearly in the areas of fixing primary health care a) Ensuring comprehensive primary Health Care (CPHC) care that has continuity with referral facilities at higher levels by an appropriate referral mechanism, b) It assures free, diagnostic and emergency services to all seeking care in public health facilities, c) targeted Infrastructure \& Human Resource Development to reach under-serviced areas throughout the country. d) It has recommendation of specific plans to scale up health services in urban areas with a focus on urban poor and establish linkages with national programs, from what was a token under-financed intervention in all previous national health plans. It also envisages achieving convergence among various departments and agencies responsible for wider determinants of health urban population e) a three- 
dimensional mainstreaming of hither to stand-alone AYUSH system of medicine for better cafeteria approach of service provision ${ }^{4}$ Currently There is skewed human resource as one sees from the figure. While $66 \%$ of population is in rural area only $33 \%$ of qualified doctors are there. On the converse the urban area has $34 \%$ of general population catered by $67 \%$ of doctors. As a result, we see $79 \%$ of urban population and $72 \%$ rural population are treated in private sector.

There may be a lot of attention on improving tertiary healthcare in India but, healthcare in rural areas remained a concern according to the Economic Survey 2018-19, tabled in the Parliament on July 4, 2019 as Sixty per cent of primary health centres (PHCs) in India have only one doctor while about five per cent have none. Gujarat emerged the worst performer, with more than 90 per cent PHCs having just one doctor. The state was followed by Kerala and Karnataka (80\% each) and Rajasthan, UP and Bihar (70 \% each). More than 10 $\%$ PHCs in Jharkhand and over $20 \%$ in Chhattisgarh function without doctors, the Survey stated. How can a health system in States with large number of PHCs functioning with just one doctor or without a doctor ensure $24 \mathrm{X} 7$ basic services? Besides the lack of doctors, participation of healthcare staff was also found to be disturbing.

The roll out plan of AB - HWCs was given as: FY 2018$19=15,000$, FY 2019-20 = 25,000 (Cumulative 40,000), FY 2020-21 = 30,000 (Cumulative 70,000), FY 2021-2022 $=40,000$ (Cumulative 1,10,000), Till 31st December 2022 $=40,000$ (Cumulative 1,50,000). Looking at the progress achieved by early 2020 one hears that against the target of 40,000 AB-HWCs by FY 2019-20, approvals for more than $62,000 \mathrm{AB}-H W C s$ were given to States/UTs and only 28005 (70\%) AB-HWC was functional as on 30.01.2020, as per ABHWC portal. We believe that the definition of functioning units may be different in different states. No wonder if most of them are on paper and at the most had changed the sign boards with no additional inputs so far [8]. At this speed it may take another 5 years to reach the target of 150,000 HWCs.

The selection process of HSCs and PHC and UPHCs for up gradation into HWCs suffers from bias of selecting the easily accessible or with political clout at least in the initial first two years. The most remote, hilly or tribal HSCs and PHCs and unrecognized urban slums did not get the opportunity of investments in Health. One would have liked $20 \%$ of HSCs that don't have their own building, $25 \%$ who do not have running water supply, one third of them who do not have electricity and $10 \%$ of them who do not have all weather roads got priority in setting up HWCS [14]. The Public sector follows "Product Centricity" strategy with Long-term focus on strengthening the product portfolio and constantly finding to new ways to expand it. It started with management of fever cases assuming that every case is case of malaria unless proved otherwise in 1953. Then came the management of diarrhoea cases through Oral Rehydration Therapy (including ORS) at community \& sub centre level in 1980 's. Later expanded to include Pneumonia management in 1990's and standalone new-born care and Integrated management of neonatal and childhood illnesses (IMNCI) at the homes and facilities in early 2000.

The NHP 2017 envisages Comprehensive Primary Health Care at Sub-centre level. Client satisfaction is the strategy followed \& all clients are treated equal. The brand equity of the public health services is poor in India according to many users and non-users. The basic doubt is about the quality of services and human behaviour of the service providers and of course irritants like long waiting time, the feeling that unless you know someone in the facility you are not cared for restrict the use of the services. But the lower middle class and poor population have no choice as the private services are not affordable. The new UHC with free comprehensive PHC and providing secondary and tertiary care through health assurance scheme will establish customer equity that is the sum of the customer's lifetime value across local customer basis. Therefore, individual care centres must have to struggle to get back the clients by not only providing available services, but also advising referral to appropriate facilities so that these customer-centric recommendations help lock in the clients for long as effectively as the quality of services themselves.

Reviewing the process of empowering para-medical workers for curative services, that started with dispensing drugs for Malaria introduced in 1953. The then malaria workers were trained to take blood smears for all fever cases during active surveillance and provide Chloroquine tablets as prophylaxis based on the age of the client and complete the recommended radical treatment if the Blood smear turned positive after microscopic examination. The concept of Multipurpose Health Workers (Male and Female) was introduced in 1974 for the delivery of preventive and promotive health care services to the community at the level of Sub-Health Centres (SHCs), the most peripheral health facilities, covering 5000 population in plains and 3000 population in hilly/ tribal/ difficult areas. The Multipurpose Health Worker (Male) was the grass root health functionary for the control of communicable diseases including Malaria, TB, Leprosy, Water Borne Diseases, as well as Environmental Sanitation, detection of disease, outbreaks and their control, health education etc. MPHW (Male) will mainly focus on activities which are related to disease, control programs, detection and control of epidemic outbreaks, environmental, sanitation, safe drinking water, first aid in emergencies like accidents, injuries, burns, etc., treatment of common/minor illnesses, communication and counselling, lifestyle diseases 
and logistics and supply management at sub-centre. In addition, he was to also facilitate ANM in MCH, Family Welfare, and Nutrition related activities. The training curriculum included 20 hours of theory and 32 hours of practical for minor ailments treatment. In view of the resurgence of Malaria and introduction of Multi-Purpose Workers (MPW) scheme in early 1974 the task of treatment of malaria cases was entrusted to ANMs also since 1974-75.

The Integrated Child Development Scheme (ICDS) was launched on 2 October 1975 in 33 (4 rural, 18 urban, 11 tribal) blocks was reinforcing the health sector human resource at the community level for complementing health services. Over the last 25 years, it was expanded progressively across the country with more than 1.3 million AWCs in early 2020. From 1975 Anganwadi centres. Set up by the department of Women and Child welfare specifically for maternal and child health care and monitoring early childhood development specially to cover most disadvantaged population. The package of services consisted of i)Complementary nutrition, ii) Vitamin A, iii) Iron and folic acid tablets, iv) Immunization, v) Health check-up, vi) Treatment of minor ailments, vii) Referral services, viii) Non-formal education on health and nutrition to women, ix) Preschool education to children 3-6 year old and $\mathrm{x}$ ) Convergence of other supportive services like water, sanitation etc. Around the same time Health ministry also recruited community volunteers and trained them for primary health care or minor ailments treatment. Diarrhoeal diseases control program launched in 1978 for better case management in 1978 lead to focus shifting to strengthening case management of diarrhoea for children under the age of 5 years and national ORT program was introduced in 19851986. In 1990 Acute Respiratory Infection Control program launching in 1990, was the real capacity building for early diagnosis and treatment for the multipurpose workers. Introduction of Integrated Management of Neonatal and childhood Illnesses (IMNCI) in 1994 with the support of UNICEF India and WHO was the real capacity building exercise for health workers in India. The scheme was expanded to entire country by 2000 , but it lost it sheen in 2008-10 mainly for want of logistics and field level support. In 2005 Accredited Social Health Activists (ASHA) were introduced for bridging the gap between health workers and community.

The new ambitious Universal health Coverage through AB-HWC (2018) plan envisages-Expanded package of primary care services-ranging from maternal and child health, communicable diseases to non-communicable diseases (universal screening, prevention, control and management of five common communicable diseases: hypertension, diabetes and three common cancers-those of the oral cavity, breast and cervix) primary health care for diseases of the eyes, oral health, ENT, mental health, provision of palliative care and care for the elderly, and medical emergencies with provision of Wide range of free drugs, Point of care diagnostics at the centres, Tele-consultation services with Medical Officers for complications, Continuum of care ensured through referral linkages and protocols, Unique health id - longitudinal health record for each individual, Services related to indigenous health system and yoga for promotion of wellness. The itemised progress can be summarized as

\section{Human Resources}

At all H\&WC's a team of at least 3 service providers $(1$ MLHP, 2/3 Multi-Purpose Workers (2 Fe \& $1 \mathrm{M}$ ) \& team of ASHAs at the norm of $1 / 1000$ population will be stationed. In urban areas, the team would consist of the MPW-F (per 10,000 pop.) and ASHAs (1/2500 pop). In most sanctioned centres the posts are created but more than $50 \%$ of the new posts are yet to be filled in.

At the strengthened $24 * 7$ PHCs having inpatient care, an additional nurse apart from IPHS standards would be posted where cervical cancer screening is being planned. In PHCs that are not envisaged to provide inpatient care, the existing nurses would receive modular training in primary care. Less than $10 \%$ of the PHCs have started screening for cancers are it cervical, breast or oral.

\section{Logistics}

To resolve more and refer less at the local levels, and to enable dispensation of medicines for chronic illnesses continued availability of essential medicines and diagnostics to support the expanded range of services will be ensured. No significant difference is visible still in the drugs logistics.

\section{Infrastructure}

It is proposed to make available sufficient space for outpatient care, pharmacies for dispensing medicines, catering diagnostic services, display of communication material and to use audio visual aids on site and space for wellness activities-the practice of Yoga \& physical exercises. Negligible additional spaces have been provided even if it be temporary on rental basis.

\section{Digitization}

HWC teams will be equipped with tablets/smart Phones to facilitate the functions of population enumeration and empanelment, recording of services delivered to enable quality follow up, referral/continuity of care and create an updated individual, family and population health profile. The gadgets will also help the local team to generate periodical reports required for monitoring at higher levels. 
ANMOL- a palm top is being tried under immunization system strengthening project with lots of starting troubles of training, maintenance, and internet connectivity issues.

\section{Use of Telemedicine/ IT Platforms}

A long term dream is to promote at all levels, teleconsultation to improve referral advice, seek clarifications, and undertake virtual training including case management support by specialists located in regional super specialty hospitals. Not yet initiated.

\section{Capacity Building}

An accredited training package in a set of primary healthcare and public health competencies for all mid-Level health providers and for other service providers to deliver the expanded range of services at HWC, combining theory and practicum with on the job training is being developed. Some states have finalized that category of people and job descriptions, recruited a few batches and they are under training as per state reports. None of such staff were seen in the field.

\section{Health Promotion}

Capacity building among community level functionaries for behaviour change communication to address lifestyle related risk factors and undertaking collective action for reducing risk exposure, improved care seeking and effective utilization of services under polio eradication and routine immunization will be expanded to cover all other interventions of UHC. Development of health promotion materials and engagement of community level workforce like Village Health Sanitation and Nutrition Committee (VHSNCs), Mahila Arogya Samiti (MAS), Self-Help Groups (SHGs) and NCC/ACC health ambassadors in schools will be strengthened. Little progress is visible as the development partners are putting together strategies and materials for health promotion or behavioural change communication.

\section{Community Mobilization}

Built on the accountability initiatives under NHM so that there is no denial of use of health care, universality and equity, community actions on social mobilization and mitigating environmental determinants, through intersectoral convergence efforts will be pursued. No concrete steps are visible in this activity.

\section{Linkages with Mobile Medical Units}

In remote and underserved areas where there is difficulty in establishing HWCs, medicines and other support could be provided to frontline workers, with the help of Mobile Medical Unit's. No progress.

\section{Referral Services}

Availability of referral care services either in Public sector or private sector varies with each illness, its care pathways and availability of specialists. For the acute illness, it is the Medical Officer in the PHC as a primary care provider and refer to appropriate level either for diagnostic facilities or for the interventions beyond what can be done at that level to the specialists in first referral units (FRUs-include Community Health Centres/ Taluka/ Sub-district or district hospitals) either physically transferring the patient or through telephonic consultation. Over next decade, states will progress to establish First Referral Units at the CHC level, and hospitals having the full complement of specialists required diagnostic and management facilities to the expanded range of services at every district headquarters. As of now referral services are available in private sector for secondary care in most of the districts towns and the super specialty hospitals at regional levels. Specific referral mechanism is yet to be established.

\section{Increased Access to Services}

HWCs would provide access to an expanded range of services indicated in Box 2 . The availability of services would evolve in different states gradually, depending on three factors- the availability of suitably skilled human resources at the HWC, the capacity at district/sub-district level to support the HWC in the delivery of that service, and the ability of the state to ensure uninterrupted supply of medicines and diagnostics at the level of HWC. States will also have the flexibility to expand the range of service to address local health problems as defined by disease prevalence. There are successful examples of organizing sickness care services under IMNCI and NBC since late 1990's and it is possible to build suitable human skills. The ability of the states to support uninterrupted supply of diagnostics, medicines and other supplies varies from state to state. Empowered Action Group (EAG) states like UP, MP, Bihar Assam may not be able to meet this goal.

On the other hand, Private sector is moving towards Customer Centric Strategy. Customer Centricity is a strategy that aligns the corporate hospitals delivers their product and services with current and future needs of their patients of a select customer to maximize their long-term profit. It is based on an understanding that not all customers are created equal. Patient centricity develops sustainable profit on the long term. The three key areas that helps are i) Customer acquisition ii) Customer retention iii) Customer development. It involves investment in the technologies, and 
human capacity necessary to collect and analyse data of their customers to meet the needs of their core customers. The other customers are also serviced but for short term, such much more numerous other clients will probably generate more profits than right customers, as they do not have to put much effort to make that profit happen. Most of the corporate hospitals especially in major cities of India are investing in such strategies i. Recognizing fundamental and inevitable differences among their customers ii. There is a quantifiable value to be found in individual clients to focus on long term marketing efforts iii. By working to quantify each customer they gain valuable insights as to how much they are willing to spend to keep existing customer and to acquire new clients iv. By doing this they serve better and in a personalized manner than their competitors [12]. By following these norms, the private health Industry is attracting lots of health tourism and some of the institution makes more profit from international clientele. There is already growing concern about some of these facilities treating the local clients as other short-term beneficiaries and giving more importance and better services to the foreigners.

\section{Essential Drugs}

Making quality medicines available at affordable prices for all, particularly the poor and disadvantaged, through exclusive outlets called "Pradhan Mantri Bhartiya Janaushadhi Kendras" is one of the best efforts made by India. 2,500 Jan Aushadhi stores will be opened in the country by end of 2020 [17]. These Kendra's sell most essential drugs for about $1 / 3$ to half the cost of the branded drugs and other supplies like sanitary pads (for INR 1). This initiative gives us a hope that soon the out of pocket expenses for the commonly used drugs and medical supplies will come down by half by 2030 .

\section{Way Forwards}

Based on personal experience of nearly 52 years in the Indian Health system having seen the system from both Government side and development partners side the author project that in best case scenario what India can achieve by 2030 by activities in the annexure 1 .

The first half a dozen of health services is being implemented for over two decades now and are well established. There is a scope to improve the outcomes in each one of them and impinge on less time of the functionaries at implementation level.

\section{Pregnancy \& Childbirth}

Percentages of pregnant women receiving pre-natal care and percentages of pregnant women receiving post-natal care are $97 \%$ and $88 \%$ respectively as per NSSO $75^{\text {th }}$ round between July 2017-June 2018 [6]. However, the quality component of the levels of anaemia and identification of Pregnancy induced hypertension are still in the range of 65$70 \%$ and need to be improved. The promotion of Institutional deliveries in the last decade country-wide has improved the proportion of skilled birth attendance to the level $91.9 \%$ in rural and $96.5 \%$ in urban India. The institutional deliveries have reached a level of $90.5 \%$ in rural and $96.1 \%$ in urban India, respectively. Only $8.1 \%$ in rural area and $3.4 \%$ in urban areas are home deliveries attended by unskilled birth attendants. The proportion of caesarean sections stands high at $38.8 \%$ in Public sector facilities and 59.9\% in private sector services that may need introspection and justification. An average cost of normal childbirth in Indian Rupees was 3746 ( $G=2084, P-12931)$ in rural and 8382 (G-2459, P-17960) in urban facilities. The caesarean section costed 20,200 (G5423, P-29406) in rural and 28058 (G-5504, P-37508) in Urban India [6].

\section{Neonatal and Infant Care}

Institutional deliveries, home based new-born care and Sick new-born care units (SNCU) have improved the newborn survival in the last 10 years. The neonatal mortality rate (NMR) declined from 52 per 1000 live births in 1990 to 28 per 1000 live births in 2013. At district level, while $9 \%$ of the districts have already reached the NMR targeted in SDG3, nearly half (315 districts) are not likely to achieve the 2030 target even if they realize the NMR reductions achieved by their own states between the last two rounds of National Family Health Survey of India [15]. That invites more efforts in neonatal care and push for the India New-born Action Plan (INAP) 2014. Full Immunization coverage among under 5 -year-old children was in the range of $57 \%-62 \%$. The difference between urban (62.1\%) and rural (58.4\%) and male $(58.5 \% 0$ and female $(60 \%)$ children was insignificant. This situation is not encouraging as despite government's efforts of intensive catch up immunization campaigns in the name of Indra-Dhanush and development partners like UNICEF, WHO and UNDP, neither the coverage has reached desired $90 \%$ level nor the routine immunization has settled. Immunization I fact has been consuming nearly $20-25 \%$ of staff time at PHC level $[16,17]$.

\section{Child and Adolescent Care}

While the growth and development of children is being monitored by the department of Women and Child welfare, the nutritional situation of children is still a matter of concern , although the prevalence of stunting among underfive children decreased from $52 \%$ to $38 \%$ and underweight declined from 53\% to 36\% between 1992 and 2016, prevalence is still alarmingly high [18]. In 2016, India had 
62 million stunted children, accounting for $40 \%$ of the global share of stunting. To address the childhood illnesses India had launched the Indian version of global IMCI called as integrated management of neonatal and childhood illnesses (IMNCI) in 2003. By June 2010, it had been implemented in 223 of India's 640 districts and more than 200000 workers had been trained. Unfortunately, it was not pursued with the rigor it deserved and demanded. Intensive on-job support and logistic supply is still wanting. It is high time that the intervention gets its due importance. There are many healthcare programs under various ministries to address the problems of adolescents, namely, Kishori Shakti Yojana, Balika Samridhi Yojana, Rajiv Gandhi Scheme for Empowerment of Adolescent Girls, "SABLA", Rashtriya Kishor Swasthya Karyakram, and Adolescent Reproductive Sexual Health Programme (ARSH). Adolescent Health Programme as an overarching Umbrella programme that covers all the health interventions for children and youth, as pursued under NHM. The outcomes of these programs need to be evaluated and requisite push given to achieve the full potential of all such programs.

\section{Family Welfare \& Reproductive Health Program}

Is one of the India's oldest national program since 1951. In 2017, Ministry of Health and Family Welfare launched "Mission Pariwar Vikas" a central family planning initiative. The key strategic focus of this initiative is on improving access to contraceptives through delivering assured services, ensuring commodity security, and accelerating access to high quality family planning services. India's fertility rate as of 2016, was 2.3 births per woman and the program's overall goal is to reduce overall fertility rate to 2.1 by the year 2025 . Two contraceptive pills, MPA (Medroxyprogesterone acetate) and Chaya are being made freely available to all government hospitals. Special focus in about 146 high fertility districts of UP, MP, Bihar, Jharkhand, Chhattisgarh, Assam, and Rajasthan should yield better outcomes.

\section{Management of Communicable Diseases}

Communicable disease is under the overall umbrella of NHM. The past two decades have seen enormous achievements in control of infectious diseases, due to sanitation and food safety, vaccines, antibiotics, and improved nutrition. Vaccination has eradicated smallpox, eradicated poliomyelitis, and has greatly reduced Whooping cough, diphtheria, tetanus, and measles. Antibiotics have helped in minimizing the damages of many tropical diseases, like malaria, tuberculosis, typhoid, Leprosy, Kala-Azar, Filariasis etc, and made possible eradication of Guinea worm disease. New diseases such as HIV and forms of influenza (H1N1, H5N1), Zika virus, Hanta virus and recently COVID 19
Pandemic, have taken both professional and population by surprise and have renewed the challenges before the public health community. Public Health in India has showed that it was a whole-of-society's approach to health that aimed at equitably to maximize the level and distribution of health and well-being focusing on people's needs and preferences early in the pandemic and ensuring the continuum from health promotion and disease prevention efforts like, containment, social distancing, hand wash, use of masks, cough etiquettes, toilet hygiene to treatment, rehabilitation and lockdown, and as close as feasible to people's everyday environment. Focus shifting to strengthening government health system by creating dedicated facilities, procuring ventilators, PPEs, and Rapid test kits, and strengthening laboratory services for PCR. Emergence of antibiotic-resistant strains of common organisms due to overuse of antibiotics and lack of vaccines for many dangerous microorganisms poses problems to humanity. This stresses the need for new vaccines, effective antibiotics and strengthened environmental control measures. New knowledge of the microbiological origins of cancers such as that of the cervix, stomach and liver have strengthened primary prevention and brought hope that new cures will be found for other chronic diseases of infectious origin. Tragically long delays in adopting "new" and cost-effective vaccines cause hundreds of thousands of preventable deaths each year in developing and mid-level developed countries [18].

\section{Management of IDSP}

Integrated disease surveillance Program was launched in India in 2004 with the support of the World Bank and WHO. It is a decentralized laboratory-based IT enabled disease surveillance system for epidemic prone diseases to monitor disease trends and to detect and respond to outbreaks in early rising phase through trained Rapid Response Team (RRTs). The concern of major hospital's poor response to transmission of weekly surveillance reports (P\&L forms) continues, though all of them contribute a lot once there is an outbreak/epidemic or a pandemic. Routine reporting from major hospitals may be made mandatory and monitored, as I feel we are missing many outbreaks in the initial stages for want of reporting from OPD cases (both P\& L forms).

The expanded services are going to pose bigger challenges to establish and give outcomes at least for next 3-5 years.

\section{Daily OPD's at HWCs}

The health centres upgraded HWCs will encounter challenges in all aspects like infrastructure building, human resource capacity building and drugs other supply logistics. 
Most importantly making services available at convenient timings of the local population, based on seasonal farming activities and gaining their trust of the population will demand not only quality services, good behaviour of the service providers and capacities of referral to the appropriate referral institutions and their responses. This calls for a referral mechanism that has not been achieved in many vertical programs like TB, Diabetes, Asthma, IMNCI, NBC, COPD etc. so far. The HWCs of present PHCs with medical officers may be able to pull through most of the activities except mental health; Initial support by a Medical officer from nearest PHC on weekly basis will help on job skill and brand building.

\section{Screening, Prevention, Control, and Management of NCDS}

Screening of Diabetes and Hypertension may be achieved at least at PHC upgraded facilities, but screening for breast, cervical and oral cancers may fall short of achievement by $50 \%$.

\section{Care for Eye and ENT}

These services may be just made available by end of 2030 at Health Sub centre turned HWCs. Even PHC turned HWCs may be able to meet a quarter of the demands and may have to refer majority of cases.

\section{Basic Oral Health Care}

Identifying carries and referral may be possible to achieve at PHC turned HWCs. Medical officer (MO) may do this task in their weekly visits even in other HWCs.

\section{Elderly Care}

Can begin in PHC turned HWCs and may meet about $25 \%$ of the problem of elderly care. MO's may take up this activity in weekly clinics.

\section{Emergency Services}

Given the fact that there is hardly one doctor in majority of the PHCs expecting round the clock emergency services will be overly ambitious. Some Emergencies during OPD hours may be managed.

\section{Screening and Management of Mental Health}

NIMHANS run district mental health program that was tried in Bellary district of Karnataka and some other states gives a hope that at least $50 \%$ of primary mental health issues can be tackled at PHC level HWCs.

\section{PIPs for CPHC}

The biggest challenge I visualize is the preparation of integrated CPHC plans for HWCs. So far neither Government of India, any states have made any efforts in this direction. This is crucial in a large country where independent vertical programs have pulled and pushed the workers in different directions periodically that confuse them so much that they can take shelter for not doing anything. The development partners like WHO, UNICEF, UNFPA and UNDP may have to take lead in this activity and create model plans for one district in each state and in the process also build state capacities for the same.

\section{Conclusion}

Although there are major challenges ahead to meet the ambitious goals of NHP 2017, it is important to capitalize on the current high level of political commitment accorded to comprehensive primary health care ${ }^{4}$. If the all available resources are mobilized just like we are doing for addressing current Pandemic of COVID 19, I am sure we will awfully close to achieve the goals of majority of intentions.

\section{References}

1. (2018) A vision for primary health care in the $21^{\text {st }}$ century, towards universal health coverage and the sustainable development goals, Technical Series on Primary Health care, UNICEF \& WHO.

2. Gangolli LV, Duggal R, Shukla A (2005) Constitutional provision of health services in India, Review of Health Care in India.

3. Origin and evolution of Primary health care in India. Health systems Development Primary Health Care, Origin and Evolution.

4. National Health Policy (2017) Ministry of Health and Family Welfare Government of India.

5. (2019) Rural Health Statistics 2018' GOI a report prepared by Government of India, Ministry of Health and Family Welfare.

6. (2019) Key indicators of Social Consumption of IndiaHealth, NSSO 75th round July2017-June 2018 NSO.

7. (2019) Persons with Disabilities in India NSS $76^{\text {th }}$ round (July-December 2018), NSO.

8. (2019) Opening of Wellness Centres under Ayushman Bharat Yojana, 29214 AB-HWC are functional as on 30.01.2020, Press Information Bureau, Government of India, Ministry of Health and Family Welfare, pp17: 38. 
9. Kaur B (2019) Economic Survey 2018-19, Down to Earth: Healthcare still inaccessible in rural India.

10. Kishanrao S (2018) Can current Indian health system achieve health related SDG's. Open J Pediatr Child Health $4(1): 13-30$.

11. Ved RR, Gupta G, Singh S (2019) India's health and wellness centres: realizing universal health coverage through comprehensive primary health care. WHO South East Asia J Public Health 8(1): 18-20.

12. (2019) Ayushman Bharat, Health and Wellness Centres, Accelerating towards health for all. Ministry of Health and Family Welfare, Government of India.

13. Sriram S (2019) Availability of infrastructure and manpower for primary health centres in a district in Andhra Pradesh, India. Journal of Family Medicine and Family Care 7(6): 1256-1262.
14. Rawat M (2019) 4 yrs. of Swachh Bharat but 38\% govt hospitals in rural India do not have staff toilets.

15. Bora JK, Saikia N (2018) Neonatal and under-five mortality rate in Indian districts with reference to Sustainable Development Goal 3: An analysis of the National Family Health Survey of India (NFHS), 20152016, PLoS ONE 13(7): e0201125.

16. (2013) National Urban Health Mission.

17. PM Jan Aushadhi Yojana, Pradhan Mantri Bhartiya Jan Aushadhi Yojana Kendra.

18. Flahault A (2018) Communicable Diseases: Achievements and Challenges for Public Health, Ursula Schlipköter, Antoine Public Health Reviews, 90-119 \& "National Centre for Disease Control" Ministry of Health \& Family Welfare, Government of India Portal 32(1). 